\title{
Drinking problems and mortality risk in the United States
}

\author{
Richard G. Rogers ${ }^{a},{ }^{\star}$, Jason D. Boardman ${ }^{a}$, Philip M. Pendergast ${ }^{a}$, and Elizabeth M. \\ Lawrence $^{\text {a }}$ \\ aDepartment of Sociology and Population Program, Institute of Behavioral Science, 483 UCB, \\ University of Colorado, Boulder, CO 80309-0483, United States
}

\begin{abstract}
Objective-We examine the links between 41 problems related to alcohol consumption and the risk of death among adults in the United States.
\end{abstract}

Method-We use Cox proportional hazards models and data from the nationally representative prospective National Health Interview Survey-Linked Mortality Files (NHIS-LMF).

Results-Drinking problems are relatively common among moderate and heavy drinkers and these problems are associated with increases in the risk of death. The strongest associations between problem drinking and mortality involved cases in which physicians, family members, or friends intervened to suggest reduced drinking. Losing one's job because of drinking problems within their lifetime (HR=1.36, 95\% confidence interval $[\mathrm{CI}]: 1.11,1.65)$ was strongly linked to mortality risk. Social risks were equally or more strongly linked to mortality than physiological consequences of alcohol abuse such as lifetime reports of needing a drink to stop shaking or getting sick (HR=1.23, 95\% CI: 1.09, 1.40). Most importantly, these associations were evident despite statistical controls for alcohol consumption levels and demographic, social, economic, behavioral, health, and geographic factors.

Conclusions-Our results highlight the independent and additive effects of alcohol-related problems and alcohol consumption levels on the risk of death. We recommend that studies examining the mortality risks of alcohol consumption take into account drinking status and also specific drinking-related problems, paying particular attention to social problems related to alcohol use or abuse.

\section{Keywords}

alcohol consumption; drinking problems; alcohol dependence; alcohol abuse; substance use disorder; mortality; NHIS

\footnotetext{
(C) 2015 Published by Elsevier Ltd.

*Corresponding author. Tel. +1 303492 2147; fax: +1 303492 2151. Richard.Rogers@ Colorado.edu (R.G. Rogers).
}

Publisher's Disclaimer: This is a PDF file of an unedited manuscript that has been accepted for publication. As a service to our customers we are providing this early version of the manuscript. The manuscript will undergo copyediting, typesetting, and review of the resulting proof before it is published in its final citable form. Please note that during the production process errors may be discovered which could affect the content, and all legal disclaimers that apply to the journal pertain. 


\section{Introduction}

Although there is extensive literature on the association between alcohol use and mortality

(Di Castelnuovo et al., 2006; Ronksley et al., 2011), few studies have examined the associations between specific alcohol-related problems and mortality. Dawson (2000a) provides some of the only evidence that alcohol dependence is linked to an increased risk of death above and beyond the level of alcohol consumption. This research is important because it shows that use and abuse, while certainly correlated with one another, denote independent pathways through which alcohol-related behaviors can influence mortality. To date, however, no existing research has examined each specific alcohol problem and corresponding DSM indicator as an independent mortality risk.

Dawson (2000a) examined the risk of death associated with alcohol dependence, classified according to the definitions given in the Diagnostic and Statistical Manual (DSM) at that time, the DSM-IV (APA, 1994), among adults aged 25 and older, using the 1988 National Health Interview Survey matched to the National Death Index (NDI) through 1995. She constructed her alcohol dependence measure from 17 of 41 alcohol-related indicators and showed that rates of alcohol dependence increased with increasing consumption; it was $9 \%$ among light drinkers, $24 \%$ among heavy drinkers, and $42 \%$ among very heavy drinkers. Compared to lifetime abstainers, dependent drinkers suffered increased risk of death. For example, moderate and dependent drinkers were $32 \%$ and very heavy and dependent drinkers were $65 \%$ more likely to die over the follow-up period. Compared to nondependent drinkers, dependent drinkers were more likely to have long and heavy drinking histories, drink greater volumes of alcohol, engage in heavy episodic drinking (HED), and have more health problems, including more major limitations, hospitalizations, and bed days (Dawson, 2000a). However, individuals can experience drinking problems without dependence. According to recent estimates, $90 \%$ of excessive drinkers are not alcohol dependent (Esser et al., 2014). Thus, calculating mortality risk for the complete list of 41 individual problems among all drinkers may capture a broader scope of drinking's health consequences.

Further, Dawson's informative results suggest that it may be useful to calculate mortality risk for the updated DSM-V, and over a longer follow-up period. The current DSM version lists 11 domains of DSM-V criteria for substance use disorder (SUD) that describe current or lifetime physiological, psychological, behavioral, and social consequences of drinking (American Psychiatric Association [APA], 2013b). While the DSM-IV defined abuse and dependence separately, the DSM-V defines one SUD with categorizations of mild, moderate, and severe. Meeting SUD criteria may indicate a cluster of drinking problems that differ from the individual problems. Health-damaging behaviors such as problem drinking earlier in life can increase risk of disease and death later in life (Graham, 2002; Kuh et al., 2003; Montez and Hayward, 2011) and many assume that the primary effects of alcohol consumption are physiological and damage specific organs. However, exposure to various risks early in life may initiate biological, psychological, and social chains of risk, which can be mediated by various factors, including socioeconomic status (Kuh et al., 2003). Problem drinking may reduce physiological or psychological resilience and increase susceptibility to death through a variety of causes, including heart disease, stroke, cancer, liver disease, chronic alcoholism, and external causes of death (suicide, homicide, motor vehicle 
accidents, and unintentional injury; Brady, 2006; Dawson, 2001, 2011; Single et al., 1999;

Skog, 2002).

In particular, social conflict related to drinking problems may be one of the main mechanisms linking excess alcohol consumption to increased mortality risk. Drinking can cause social problems at home, school, and work, and with social and sports activities (Ames et al., 1997), family, friends, and coworkers (Barnes and Farrell, 1992), and the legal system (Caetano, 1997). Social resources-including ties to family, friends, school, work, and the community - protect against the risk of death (Berkman and Syme, 1979; Seeman, 1996). Thus, social disruptions such as the end of a relationship, decreased social interactions, or the loss of support from colleagues because of alcohol-related problems may be mechanisms through which chronic alcohol use leads to elevated risk of death. Furthermore, drinking is linked to emotional problems, depression, and lack of personal control (Holahan et al., 2003; Nigg et al., 2006; Windle and Windle, 1996).

There are also beneficial physiological and psychological effects of light to moderate consumption. Light to moderate alcohol consumption can enhance mood, reduce stress, lower anxiety, and decrease the risk of depression, which can in turn contribute to a stronger sense of sociability, social integration, and cohesion (Peele and Brodsky, 2000). Light to moderate alcohol consumption can also reduce the risk of heart disease through increased levels of HDL cholesterol, apolipoprotein A1, and adiponectin, and reduced levels of fibrinogen (Agarwal, 2002; Brien et al., 2011). Because low and even moderate levels of alcohol have been linked to salutary health profiles in previous research, it is important to control for current and past drinking levels to examine the influence of specific alcohol problems above and beyond current drinking levels.

In identifying the relationships between drinking problems and mortality, this study seeks to determine whether drinking's health consequences depend on the social consequences of drinking (in addition to consumption patterns). This paper addresses four central aims. First, we describe the prevalence of specific drinking problems across drinking statuses and consumption levels among a representative sample of U.S. adults. Second, to examine the association between specific drinking problems and mortality, we are the first to use information on 41 specific alcohol-related problems from adults in 1988 and their subsequent mortality nearly 20 years later. Third, we examine the association between clusters of drinking problems, as defined by the DSM-V criteria, and mortality. Finally, we determine the mediating effects of SUD on the association between drinking status and mortality.

\section{Methods}

\subsection{Data}

The National Institute on Alcohol Abuse and Alcoholism sponsored the 1988 National Health Interview Survey (NHIS) Alcohol Supplement, a nationally representative survey of the noninstitutionalized population focusing on alcohol use and related problems experienced over the past year (NCHS, 1989, 2010). The Alcohol Supplement contains 
43,809 adults aged 18 and over. Because our focus is on current legal drinkers, we limit our analyses to individuals with known survival status who are aged 21 and above.

The 1988 NHIS Alcohol Supplement allows us to investigate the mortality risk of 41 drinking problems. The questions from this supplement classifies individuals as current drinkers (12 drinks in the last year), former drinkers (12 drinks in a previous year but not in the last year), lifetime abstainers (less than 12 drinks in lifetime), and lifetime infrequent drinkers (less than 12 drinks in any one year). The supplement asked current drinkers about problems in the last 12 months, yielding 20,748 respondents for current problems, and current and former drinkers whether they had experienced each problem in their lifetime, resulting in 28,542 respondents for lifetime problems. We exclude lifetime infrequent drinkers and lifetime abstainers, except some analyses which use lifetime abstainers as a reference group for current drinkers. We also exclude 1,051 individuals from our analyses because they did not respond to questions on the frequency or the occasion-specific volume of their drinking over the past year, which prevented us from determining the average volume of drinks they consumed. The analytic sample is thus 19,697 for problems in the last year and 27,491 for lifetime problems. Models including current drinkers $(\mathrm{N}=19,697)$ and lifetime abstainers $(\mathrm{N}=7,859)$ produce a sample size of 27,556 .

In 2010, the National Center for Health Statistics (NCHS) linked the NHIS to the National Death Index (NDI) through probabilistic record matching to identify individuals who died between the time of the interview and the end of 2006. NHIS collects all of the 13 NDI matching variables, including social security number; first and last name; middle initial; day, month, and year of birth; sex; race; father's surname; state of birth and residence; and marital status. We drop less than $1 \%$ of records that do not include enough information for accurate record linkage, termed ineligible, from our analyses, and then incorporate the new NCHSproduced weights for the eligible sample (NCHS, 2009). Of the past year problem and lifetime problem analytic samples, 3,365 and 5,836 adults died within this period, respectively.

This dataset is ideal for our research in three ways. First, it contains detailed information from a large, representative sample related to alcohol consumption and problem drinking behaviors. Second, these problem behaviors align well with the DSM-V criteria for SUD, allowing us to investigate the relationship between clinically defined alcohol dependency and mortality. Third, the relatively long follow-up exposes potential lagged effects of alcohol use and problem behaviors on the risk of death (Rehm et al., 2010).

\subsection{Methods}

2.2.1 Alcohol consumption and problem drinking-For current drinkers, we calculate alcohol volume (drinks per day on the days that individuals drink multiplied by the number of days that individuals drink per year, divided by 366 [because 1988 was a leap year]) from self-reports of current drinkers, which studies have generally found to be reliable and valid (Del Boca and Darkes, 2003). We follow Breslow and Graubard's (2008) coding strategy, which groups drinkers into four categories based on volume: those who drink less than 1 drink, 1 to less than 2, 2 to less than 3, and 3 or more drinks per day, on average. Former drinkers are included together in one category. 
NCHS asked current drinkers how many times in the past 12 months they had experienced 41 different problems with drinking (see Chyba and Washington, 1993). To examine the mortality risk of individual problems, we dichotomize each problem, coding those reporting one or more times as 1 and those reporting none or never as 0. For problems ever experienced in one's lifetime, current and former drinkers responded whether they had (coded 1) or had not (coded 0 ) experienced each of the 41 problems.

We followed the method of Dawson (2000a) to operationalize the 11 criteria for DSM-IV alcohol abuse and dependence, then updated the measures to be consistent with the 11 DSM$\mathrm{V}$ criteria for SUD. The newly-released DSM-V drops legal problems and drinking for relief from diagnostic criteria, adds new criteria for craving and social consequences of drinking, and no longer distinguishes between abuse and dependence as it groups together all SUDs. It does specify severity, categorizing disorders as mild, moderate, or severe according to the number of criteria met by subjects (APA, 2013a). We operationalized the new "craving" criterion by using responses to three questions: In the past 12 months how many times have you (1) Needed a drink so badly you couldn't think of anything else? (2) Felt uneasy if alcohol was not around in case you wanted a drink? (3) Had a strong desire or urge to drink? A respondent who had experienced any of these problems at least twice in the past year was classified as having met the "cravings" criterion. The "social" criterion was operationalized using two questions: In the past 12 months how many times have you (1) Had a spouse or someone you live with threaten to leave you? (2) Had family, friends, or coworkers suggest that you stop or cut down on your drinking? Respondents experiencing either problem at least twice in the past year fulfilled the "social" criterion.

The criteria that remained consistent with the DSM-IV were considered fulfilled if the respondent had experienced one or more problems within the relevant group at least twice in the past year, with two exceptions: at least three positive symptoms of withdrawal were required, with at least two being experienced twice in the past year, and a minimum of two tolerance symptoms were required, with no duration criterion. Finally, following the new APA guidelines (2013a), we categorized current drinkers as having no (0-1 criteria), mild (2-3 criteria), or moderate to severe (4 or more criteria) SUD. Because the NHIS did not ask about the frequency of lifetime problems, we calculate DSM criteria among current drinkers only and assume lifetime abstainers have no SUD. Table 1 provides additional information regarding the construction of the SUD measure.

2.2.2 Control variables-We control for a variety of factors that may mediate the association between drinking and mortality (see Himes, 2011), including sex (female=referent), race/ethnicity (non-Hispanic white [referent], non-Hispanic black, Hispanic, Asian, and other), marital status (married [referent], never married, divorced, and widowed), socioeconomic status (education and income), cigarette smoking status (never smoked [referent], former smoker, and current smoker), body mass index (BMI), and region of residence (Northeast [referent], Midwest, South, and West). Education is the number of years of education completed. Income is logged to account for diminishing returns on survival with increasing income. We treat BMI as a continuous measure. We control for BMI because it has been associated with alcohol consumption and identified as a major risk factor for overall mortality, and is one of the very few if only physiological measurements 
available in the data set. Because drinking and smoking are interrelated (Dawson, 2000b; Rogers et al. 2013), it is important to control for the effects of cigarette consumption. Drug use and mental health conditions may also be related to drinking, but were not included in the dataset.

We found no strong or consistent sex differences in the relationships between drinking problems and mortality, even though compared to females males report higher prevalence rates of drinking and generally drink larger amounts of alcohol. Male and female prevalence rates of drinking problems across consumption rates are available upon request. Thus, we control for but do not disaggregate our results by sex. Auxiliary analyses (available upon request) tested for but did not find significant direct or mediating effects of HED on the associations among drinking problems, DSM diagnosis, drinking status, and mortality, and thus it is excluded for parsimony. We measured HED as the number of days the respondent reports drinking 5 or more drinks in the past year (Jackson 2008).

2.2.3 Multivariate models-We use Cox proportional hazards models to examine the risk of overall mortality associated with problem drinking. We do not present cause-specific mortality results because the models did not reveal any new insights that were not already captured by all-cause mortality. Duration is the time to death or 2006 and entry is the age at interview. This approach implicitly accounts for age and is the preferred approach to specifying duration in survival models (see Singer and Willet, 2003; Thiebault and Benichou, 2004). NHIS top-codes individuals over the age of 99 and we drop individuals under 21, resulting in an age range from 21 to 99 . To determine the independent effect of each drinking problem, and because drinking problems are interrelated, Table 3 provides hazard ratios for each drinking problem separately. Thus, we compare those who reported to those who did not report difficulty stopping drinking, for example. These associations may understate risk, given that models look at individual problems, and respondents reporting one problem may have experienced other problems.

Duration effects may be less important for alcohol dependence than for other health conditions, because much research documents that alcoholics struggle with alcohol throughout their lives. Thus, it is quite reasonable to expect that alcohol-related problems reported at the time of the interview could contribute to increased mortality risk years later.

We use multiple imputation methods to deal with missing data because they provide unbiased and more efficient estimates than other missing data approaches, including listwise deletion, provided that data are missing at random or missing completely at random (Allison, 2002). We use the mi package with the "force" option in Stata 12.0 (StataCorp, 2011) to multiply-impute values missing on education, income, BMI, marital status, smoking status, each of the drinking problems, and values for the DSM-V criteria. For the sample of 19,697 current drinkers, we imputed 4.2-4.9\% for each of the reported drinking problems, $4.7-5.4 \%$ for each of the DSM-V criteria, $0.2 \%$ for education, $10.0 \%$ for income, $1.0 \%$ for BMI, and $0.1 \%$ for marital and smoking statuses. Imputation rates are similar for the other samples. All model estimates were based on 10 imputations, which provide good efficiency with less than 30\% missing data (Rubin, 1987; Schafer and Olsen, 1998). Comparing results from imputations with those from analyses using only observations with 
complete information revealed that the substantive findings and conclusions of this study are not sensitive to imputation. The results are very similar, with some ratios showing slightly reduced magnitude or significance, likely due to the smaller sample size.

We adjust for the complex survey design, including the eligibility-adjusted sample weights (NCHS, 2009), through the "svy" package in Stata (StataCorp, 2011). Analyses follow variance estimation procedures for 1985-1994 NHIS data provided on the NCHS website (NCHS, 2013). We tested and confirmed that we are not violating the proportionality assumption by plotting Kaplan-Meier curves for several focal independent variables (figures not shown but available upon request) and conducting tests of proportionality.

Proportionality results indicated that, although models failed global tests of proportionality, our main variables of interest did not violate proportionality, as only 1 of 41 lifetime problems, 9 of 41 past year problems, and no drinking status variables were significant at the .05 level. We also conducted sensitivity analyses, examining the effect of shortening the duration of our follow-up periods from the initial 18 years to 15 and 10 years, and excluding individuals who died in the year of the interview (results not shown). These auxiliary models showed similar but generally stronger results from those presented in the manuscript, which suggests that our results may be a conservative representation of the effects of drinking problems on mortality. We prefer the longer follow-up period because it produces more stable estimates and smaller standard errors.

\section{Results}

Table 2 displays the prevalence of specific drinking problems among current drinkers (Panel A), and among current and former drinkers who report drinking problems within their lifetime (Panel B). For ease in interpretation, we have grouped these problems within the 11 DSM criteria, in bold. Perhaps most striking, although many specific drinking-related problems are uncommon, it is quite common for drinkers to experience at least one drinkingrelated problem in the previous year. For example, even $48 \%$ of light drinkers experienced at least one drinking-related problem over the past year.

Still, there is substantial variation in these problems. For instance, among all current drinkers, 23\% started drinking even though they did not intend to, 20\% drank longer than intended, and one-quarter experienced a strong urge to drink. Each problem displays a gradient, with the percentage increasing with consumption level. For example, people who experienced a strong urge to drink over the previous year were $19 \%$ of light, $40 \%$ of moderate, $50 \%$ of moderate-to-heavy, and $57 \%$ of heavy drinkers. Fortunately, many problems are rare, such as losing a job or having a spouse threaten to leave because of drinking. However, the magnitude of the differences by consumption level can be substantial. For instance, only $1 \%$ of light but $13 \%$ of heavy drinkers had been arrested because of drinking.

A larger percentage of current drinkers report problems over their lifetimes compared to the previous year (see Panel B). For instance, the percentage of current light drinkers who reported driving a car after drinking too much was $36 \%$ over their lifetime, but just $12 \%$ in the past year. In many instances, former drinkers reported more problems than light drinkers

Drug Alcohol Depend. Author manuscript; available in PMC 2016 June 01. 
over their lifetimes, which may explain their drinking cessation. This difference between current and former drinkers is quite pronounced among those within the "Continue" category. For instance, $8 \%$ of former drinkers, but just 3\% of current light drinkers, reported that drinking threatened their health. We expect that the tremendous variations in drinking problems by consumption level revealed in Table 2 will translate into sizeable differences in mortality risk.

Table 3 presents the risk of death for each of the specific problems. We estimate each problem separately. Because there are multiple models, readers should interpret significance across the 41 problems carefully; nevertheless, the number of significant associations far exceeds what we would expect due to chance alone. Indeed, almost half of the individual drinking problems reported in the previous year were significantly associated with heightened mortality risk over the follow-up period, net of the effects of alcohol consumption levels, age, sex, race and ethnicity, marital status, education, income, smoking, BMI, and region. For instance, current drinkers who found it difficult to stop drinking once they started experienced 15\% higher risk of death over the follow-up period, those who cut down on other social and sports activities because of drinking had a $46 \%$ higher risk of death, and those whose doctors suggested that they reduce their drinking experienced $67 \%$ higher risk, compared to those who did not report these problems. Many problem drinking behaviors that do not map onto the DSM criteria were also related to substantially increased mortality, including losing one's job because of drinking ( $\mathrm{HR}=1.67,95 \%$ confidence interval [CI]: 1.02, 2.75), going on benders (HR=1.54, 95\% CI: 1.07, 2.22), and attending Alcoholics Anonymous (AA) meetings (HR=1.45, CI: 1.04, 2.02).

Current and former drinkers who reported drinking problems within their lifetime also experienced higher risks of death than those who did not. Of the 41 problems, 29 were significantly associated with an increased mortality risk even after adjusting for drinking status, consumption levels (for current drinkers), and other covariates. Though more lifetime than previous-year problems carried significant mortality risks (perhaps because of the relatively larger sample size), the lifetime ratios were mostly smaller than or similar in magnitude to the previous-year ratios. For example, current drinkers who had difficulty stopping drinking in the past year showed a 15\% increased risk, compared to $12 \%$ for current and former drinkers who had ever experienced this problem. Few lifetime problems showed greater risk than the equivalent previous-year problem, though "lost ties with family and friends" and "spouse threatened to leave" show stronger and more significant effects for the lifetime groups, perhaps because these problems captured particularly risky habits for former drinkers.

Hazard ratios in Table 4 indicate the mortality risk associated with fulfillment of each of the 11 DSM-V criteria for SUD, with significant differences in 5 of the 11 categories. For example, compared to drinkers who did not report an alcohol tolerance, those who reported a tolerance experienced $24 \%$ higher risk of death over the follow-up period. Those who did not complete their major role responsibilities had $29 \%$ higher risk of mortality, and those experiencing social costs related to alcohol use had $57 \%$ higher risk of mortality over the follow-up period.

Drug Alcohol Depend. Author manuscript; available in PMC 2016 June 01. 
Table 5 shows the mortality risk associated with meeting the DSM-V criteria for mild or moderate to severe SUD. These analyses compare current drinkers to lifetime abstainers (individuals who have had fewer than 12 drinks in their lives). Compared to no SUD, moderate to severe SUD increases the risk of death by $47 \%$ over the follow-up period. The DSM indicators modestly attenuate the effects of each drinking status. For example, controlling for SUD attenuates the risk of death associated with heavy drinking by $22 \%$ $([\ln (1.47-\ln (1.35)) / \ln (1.47) * 100]$; compare Models 1 and 2). Additional models (not shown) interacted drinking status with DSM diagnoses; none were significant, indicating that the measures have independent effects on mortality. We also examined the sensitivity of our results to HED by estimating a separate model that included HED as a control (not shown); this specification did not change the results in Model 2, suggesting that the increased mortality risk of SUD is not operating through episodic periods of heavy drinking.

\section{Discussion}

Drinking problems characterize the style of alcohol consumption that is related to but distinct from their drinking status and average consumption. Our results show that current drinkers who had experienced problems related to drinking over the previous year, and current and former drinkers who had ever experienced such problems in their entire lives, suffered higher risk of death than individuals who had not experienced drinking problems.

Many current drinkers, even light drinkers, report drinking-related problems including uncontrollable drinking, a preoccupation with drinking, reduced activities because of drinking, prolonged misuse of alcohol, and withdrawal symptoms associated with drinking. As one would expect, there is a strong graded relationship between drinking status and drinking-related problems, with higher prevalence rates of drinking-related problems among individuals who report higher levels of alcohol consumption. For example, it is much more common for heavy than light drinking to be associated with losing a job.

Alcohol consumption is multidimensional, with strong biological, physiological, psychological, and social components. Interestingly, the physically hazardous problems (such as driving while drunk, or doing things that could hurt oneself or someone else) did not show a significant mortality risk, while social and psychological problems did. Problem drinking may increase mortality risk in part through lost social ties, relationships, and networks; lost income, educational, and employment opportunities; and legal and criminal problems (Ames et al., 1997; Barnes and Farrell, 1992; Caetano, 1997). Additionally, problem drinking can contribute to emotional problems including depression and loss of personal control (Holahan et al., 2003; Nigg et al., 2006; Windle and Windle, 1996).

Some of the problems that we identified among light drinkers may be directly related to light drinking, but others may be due to atypical drinking, including HED (see Dawson, 2011). A relatively high percentage of light drinkers reported that they drank more than they intended on at least one occasion in the past year. One risk of light drinking may be that it can contribute to occasional heavy drinking. Such risks could be much higher among current regular drinkers than among infrequent or former drinkers, or abstainers. Nevertheless, our

Drug Alcohol Depend. Author manuscript; available in PMC 2016 June 01. 
controls for HED did not explain this association between light drinking and drinking more than one intended (results not shown).

The association between attending AA meetings and mortality may reflect that individuals who reported attending a meeting in the past year could have been recently diagnosed as alcoholics or are more likely to have severe health, social, or legal problems that require AA attendance, including legally-mandated attendance. We also observed a substantial increase in mortality risk associated with AA if smoking status is not controlled for (results not shown), indicating that those who attend AA may be likely to have other substance dependence issues that are also closely tied to increased mortality. A primary goal of these groups should be reducing dependence on all of these substances if they aim to encourage healthy behavior and long life.

We classified alcohol abuse and dependence according to the recently released DSM-V (APA, 2013b), which classifies subjects along a spectrum of SUD (none, mild, moderate, or severe). We found significantly higher mortality risk among individuals with moderate to severe SUD, with DSM-V diagnoses modestly attenuating the effects of heavy alcohol consumption. Many problem-drinking behaviors-including losing one's job due to drinking, feeling as if one needs a drink badly, going on benders, and attending AA meetings - substantially increased mortality but do not map onto any version of the DSM criteria, suggesting that alcoholism diagnosis does not capture the complete array of harmful problem-drinking behaviors.

The association between each of the drinking statuses and mortality is partially diminished once we control for SUD. This indicates that the mortality risk associated with each drinking status, including heavy drinking, may be slightly overstated for drinkers who have not experienced problems. Nevertheless, our results indicate that drinking can be hazardous to one's health and longevity because of the problems these behaviors can engender- $87 \%$ of individuals who drank three or more alcoholic drink per day on average reported at least one drinking problem in the past year (see Table 2).

Our findings diverge from those of Dawson (2000a), who used the same Alcohol Supplement and concluded that DSM-IV-defined alcohol dependence nullified the protective effect of light and moderate drinking. In contrast, we find that the protective effect of light drinking persists even after controlling for SUD. Dawson (2000a) had different specifications - notably, she restricted the ages to 25 and older rather than 21 and older, controlled for poor health at baseline, used a shorter mortality follow-up period, and included lifetime infrequent drinkers as well as former drinkers in the analysis-which helps explain differences in the results.

This study benefits from a nationally representative sample, more detailed information on alcohol consumption and problem drinking, and a significantly longer follow-up period than previous work in this area. Still, our study is limited by the use of an older sample that does not include time-varying covariates. Although the prevalence of drinking problems may differ between 1988 and today, we contend that the mortality risks for specific problems remain quite similar. We analyze drinking status and drinking problems reported at the time

Drug Alcohol Depend. Author manuscript; available in PMC 2016 June 01. 
of the survey, but do not have information about whether respondents experienced additional (or fewer) drinking problems at a later date. Nonetheless, unlike other variables, some drinking problems may remain relatively stable over time. Any change in lifetime drinking problems should be unidirectional; additional years of life cannot erase previous drinking problems but can expose individuals to repeat or new problems.

Our results underscore the importance of drinking problems to the risk of death. Researchers and policymakers should consider multiple dimensions of alcohol consumption, including drinking status, consumption levels, and, importantly, problem drinking. Alcohol consumption has strong biological, physiological, psychological, and social components. Problems associated with alcohol consumption provide great insight into important health behaviors that are strongly related to survival chances.

\section{References}

Agarwal DP. Cardioprotective effects of light-moderate consumption of alcohol: a review of putative mechanisms. Alcohol Alcohol. 2002; 37:409-415. [PubMed: 12217928]

Allison, PD. Missing Data. Sage; Thousand Oaks, CA: 2002.

American Psychiatric Association. Diagnostic And Statistical Manual Of Mental Disorders. 4th. American Psychiatric Association; Washington, DC: 1994.

American Psychiatric Association. Washington, DC: 2013a. Highlights Of Changes From DSM-IVTR To DSM-5. Available at http://www.dsm5.org/Documents/changes\%20from\%20dsm-iv-tr \%20to\%20dsm-5.pdf Retrieved February 11, 2015

American Psychiatric Association. Washington, DC: 2013b. News release: American Psychiatric Association releases DSM-5. Available at (http://www.psychiatry.org/File\%20Library/Advocacy \%20and\%20Newsroom/Press\%20Releases/2013\%20Releases/13-31-DSM-5-Publication-NewsRelease.pdf) Retrieved February 11, 2015

Ames GM, Grube JW, Moore RS. The relationship of drinking and hangovers to workplace problems: an empirical study. J Stud Alcohol Drugs. 1997; 58:37-47.

Barnes GM, Farrell MP. Parental support and control as predictors of adolescent drinking, delinquency, and related problem behaviors. J Marriage Fam. 1992; 54:763-776.

Berkman LF, Syme SL. Social networks, host resistance, and mortality: a nine-year follow-up study of Alameda County residents. Am J Epidemiol. 1979; 109:186-204. [PubMed: 425958]

Brady J. The association between alcohol misuse and suicidal behavior. Alcohol Alcohol. 2006; 41:473-478. [PubMed: 16891335]

Breslow RA, Graubard BI. Prospective study of alcohol consumption in the United States: quantity, frequency, and cause-specific mortality. Alcohol Clin Exp Res. 2008; 32:513-521. [PubMed: 18215212]

Brien SE, Ronksley PE, Turner BJ, Mukamal KJ, Ghali WA. Effect of alcohol consumption on biological markers associated with risk of coronary heart disease: systematic review and metaanalysis of interventional studies. Br Med J. 2011; 34210.1136/bmj.d636

Caetano R. Prevalence, incidence and stability of drinking problems among whites, blacks and Hispanics: 1984-1992. J Stud Alcohol Drugs. 1997; 58:565-572.

Chaloupka HS, Grossman M. Alcohol-control policies and motor-vehicle fatalities. J Legal Stud. 1993; 22:161-186.

Chyba MM, Washington LR. Questionnaires from the National Health Interview Survey, 1985-99. Vital Health Stat. 1993; 1:1-412.

Dawson DA. Alcohol consumption, alcohol dependence, and all-cause mortality. Alcohol Clin Exp Res. 2000a; 24:72-81. [PubMed: 10656196]

Dawson DA. Drinking as a risk factor for sustained smoking. Drug Alcohol Depend. 2000b; 59:235249. [PubMed: 10812284]

Drug Alcohol Depend. Author manuscript; available in PMC 2016 June 01. 
Dawson DA. Alcohol and mortality from external causes. J Stud Alcohol. 2001; 62:790-797. [PubMed: 11838916]

Dawson DA. Defining risk drinking. Alcohol Res Health. 2011; 34:144-156. [PubMed: 22330212]

Del Boca FK, Darkes J. The validity of self-reports of alcohol consumption: state of the science and challenges for research. Addiction. 2003; 98(Suppl. 2):1-12. [PubMed: 14984237]

Di Castelnuovo A, Costanzo S, Bagnardi V, Donati MB, Iacoviello L, de Gaetano G. Alcohol dosing and total mortality in men and women. Arch Intern Med. 2006; 166:2437-2445. [PubMed: 17159008]

Esser MB, Hedden SL, Kanny D, Brewer RD, Gfroerer JC, Naimi TS. Prevalence of alcohol dependence among US adult drinkers, 2009-2011. Prev Chronic Dis. 2014; 11:1-11.

Graham H. Building an inter-disciplinary science of health inequalities: the example of lifecourse research. Soc Sci Med. 2002; 55:2005-2016. [PubMed: 12406467]

Himes, CL. Relationships among health behaviors, health, and mortality. In: Rogers, RG.; Crimmins, EM., editors. International Handbook Of Adult Mortality. Springer; New York: 2011. p. 289-310.

Holahan CJ, Moos RH, Holahan CK, Cronkite RC, Randall PK. Drinking to cope and alcohol use and abuse in unipolar depression: a 10-year model. J Abnorm Psychol. 2003; 112:159-165. [PubMed: 12653424]

Jackson KM. Heavy episodic drinking: determining the predictive utility of five or more drinks. Psychol Addict Behav. 2008; 22:68-77. [PubMed: 18298232]

Kuh D, Ben-Shlomo Y, Lynch J, Hallqvist J, Power C. Life course epidemiology. J Epidemiol Community Health. 2003; 57:778-783. [PubMed: 14573579]

Montez, J.; Hayward, M. Early life conditions and later life mortality. In: Rogers, RG.; Crimmins, EM., editors. International Handbook Of Adult Mortality. Springer; New York: 2011. p. 185-204.

National Center for Health Statistics(NCHS). National Health Interview Survey, 1988. Hyattsville, Maryland: 1989. Public Use Data Tape Documentation. Part II - Interviewer's Manual, HIS-100. Available at ftp://ftp.cdc.gov/pub/Health_Statistics/NCHS/Dataset_Documentation/NHIS/1988/ frmanual.pdf Retrieved February 11, 2015

National Center for Health Statistics. Hyattsville, Maryland: 2009. The National Health Interview Survey (1986-2004) Linked Mortality Files, Mortality Follow-Up Through 2006: Matching Methodology. (Available at http://www.cdc.gov/nchs/data/datalinkage/ matching_methodology_nhis_final.pdf) Retrieved February 11, 2015

National Center for Health Statistics. Hyattsville, Maryland: 2010. Comparative Analysis Of The NHIS Public-Use And Restricted-Use Linked Mortality Files: 2010 Public-Use Data Release. Available at http://www.cdc.gov/nchs/data/datalinkage/nhis_mort_compare_2010_final.pdf Retrieved February 11, 2015

National Center for Health Statistics. Variance Estimation For The 1985-94 NHIS Public Use Person Data. 2013. Available at http://www.cdc.gov/nchs/data/nhis/8594var.pdf Retrieved February 11, 2015

National Institute on Alcohol Abuse and Alcoholism. Alcohol Use Disorder: A Comparison Between DSM-IV And DSM-5. U.S. Government Printing Office; Washington, DC: 2013. NIH Publication No. 13-7999

Nigg JT, Wong MM, Martel MM, Jester JM, Puttler LI, Glass JM, Adams KM, Fitzgerald HE, Zucker RA. Poor response inhibition as a predictor of problem drinking and illicit drug use in adolescents at risk for alcoholism and other substance use disorders. J Am Acad Child Adolesc Psychiatry. 2006; 45:468-475. [PubMed: 16601652]

Peele S, Brodsky A. Exploring psychological benefits associated with moderate alcohol use: a necessary corrective to assessments of drinking outcomes? Drug Alcohol Depend. 2000; 60:221247. [PubMed: 11053757]

Rehm J, Baliunas D, Borges GLG, Graham K, Irving H, Kehoe T, Parry CD, Patra J, Popova S, Poznyak V, Roerecke M, Room R, Samokhvalov AV, Taylor B. The relation between different dimensions of alcohol consumption and burden of disease: an overview. Addiction. 2010; 105:817-843. [PubMed: 20331573]

Rogers RG, Krueger PM, Miech R, Lawrence EM, Kemp R. Nondrinker mortality risk in the United States. Popul Res Policy Rev. 2013; 32:325-352. [PubMed: 25045194] 
Ronksley PE, Brien SE, Turner BJ, Mukamal KJ, Ghali WA. Association of alcohol consumption with selected cardiovascular disease outcomes: a systematic review and meta-analysis. Br Med J. 2011; 34210.1136/bmj.d671

Rubin, DB. Multiple Imputation For Nonresponse In Surveys. J. Wiley \& Sons; New York: 1987.

Schafer JL, Olsen MK. Multiple imputation for multivariate missing-data problems: a data analyst's perspective. Multivariate Behav Res. 1998; 33:545-571.

Seeman TE. Social ties and health: the benefits of social integration. Ann Epidemiol. 1996; 6:442-451. [PubMed: 8915476]

Singer, JD.; Willett, JB. Applied Longitudinal Analysis: Modeling Change And Event Occurrence. Oxford University Press; New York, NY: 2003.

Single E, Robson L, Rehm J, Xi X. Morbidity and mortality attributable to alcohol, tobacco, and illicit drug use in Canada. Am J Public Health. 1999; 89:385-390. [PubMed: 10076491]

Skog OJ. Alcohol consumption and mortality rates from traffic accidents, accidental falls, and other accidents in 14 European countries. Addiction. 2002; 96:49-58.

StataCorp. Stata Statistical Software: Release 12. StataCorp; College Station, TX: 2011.

Thiébaut A, Bénichou J. Choice of time-scale in Cox's model analysis of epidemiologic cohort data: a simulation study. Stat Med. 2004; 23:3803-3820. [PubMed: 15580597]

Windle M, Windle RC. Coping strategies, drinking motives, and stressful life events among middle adolescents: associations with emotional and behavioral problems and with academic functioning. J Abnorm Psychol. 1996; 105:551-560. [PubMed: 8952188]

Drug Alcohol Depend. Author manuscript; available in PMC 2016 June 01. 


\section{Highlights}

- It is common for moderate and heavy (and even light) drinkers to experience at least one drinking-related problem in the previous year.

- Drinking problems reported over the past year and over a person's lifetime increase the risk of death, net of covariates, including current drinking status.

- Current drinkers who reported that in the previous year they had started drinking even though they hadn't intended to on at least one occasion experienced $13 \%$ higher risk of death over the follow-up period.

- Individuals who had been arrested in the previous year because of drinking experienced $37 \%$ higher risk of death over the follow-up period.

- Researchers and policymakers should consider multiple dimensions of alcohol consumption, including drinking status, consumption levels, and, importantly, problem drinking. 


\section{Table 1}

\section{"Diagnostic criteria for DSM-5 SUD with abbreviations in parentheses.}

"Alcohol is often taken in larger amounts or over a longer period than was intended" [Larger]

- "There is a persistent desire or unsuccessful efforts to cut down or control alcohol use" [Cut down]

- "A great deal of time is spent in activities necessary to obtain alcohol, use alcohol, or recover from its effects" [Time spent]

- "Recurrent alcohol use resulting in a failure to fulfill major role obligations at work, school, or home" [Major role]

- "Recurrent alcohol use in situations in which it is physically hazardous" [Hazard]

- "Important social, occupational, or recreational activities are given up or reduced because of alcohol use" [Give up]

- "Alcohol use is continued despite knowledge of having a persistent or recurrent physical or psychological problem that is likely to have been caused or exacerbated by alcohol" [Continue]

- "A need for markedly increased amounts of alcohol to achieve intoxication or desired effect" or "a markedly diminished effect with continued use of the same amount of alcohol" [Tolerance]

- "The characteristic withdrawal syndrome for alcohol" or "alcohol (or closely related substance, such as benzodiazepine) is taken to relieve or avoid withdrawal symptoms" [Withdrawal]

- $\quad$ "Continued alcohol use despite having persistent or recurrent social or interpersonal problems caused or exacerbated by the effects of alcohol" [Social]

- "Craving, or a strong desire or urge to use alcohol" [Cravings]

* Reprinted by permission of the National Institute of Alcohol Abuse and Alcoholism (2013, p. 2]. 


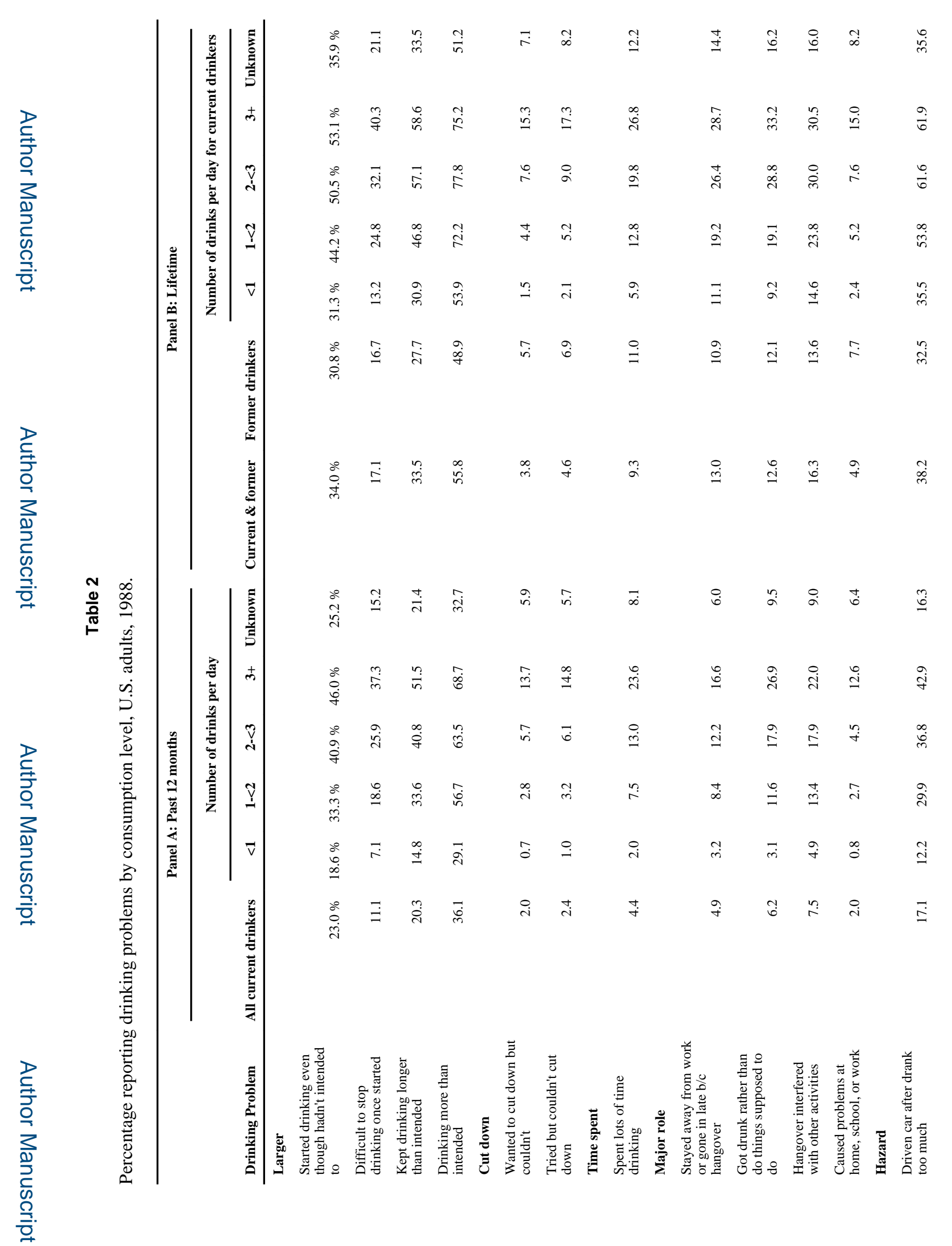

Drug Alcohol Depend. Author manuscript; available in PMC 2016 June 01. 
Rogers et al.

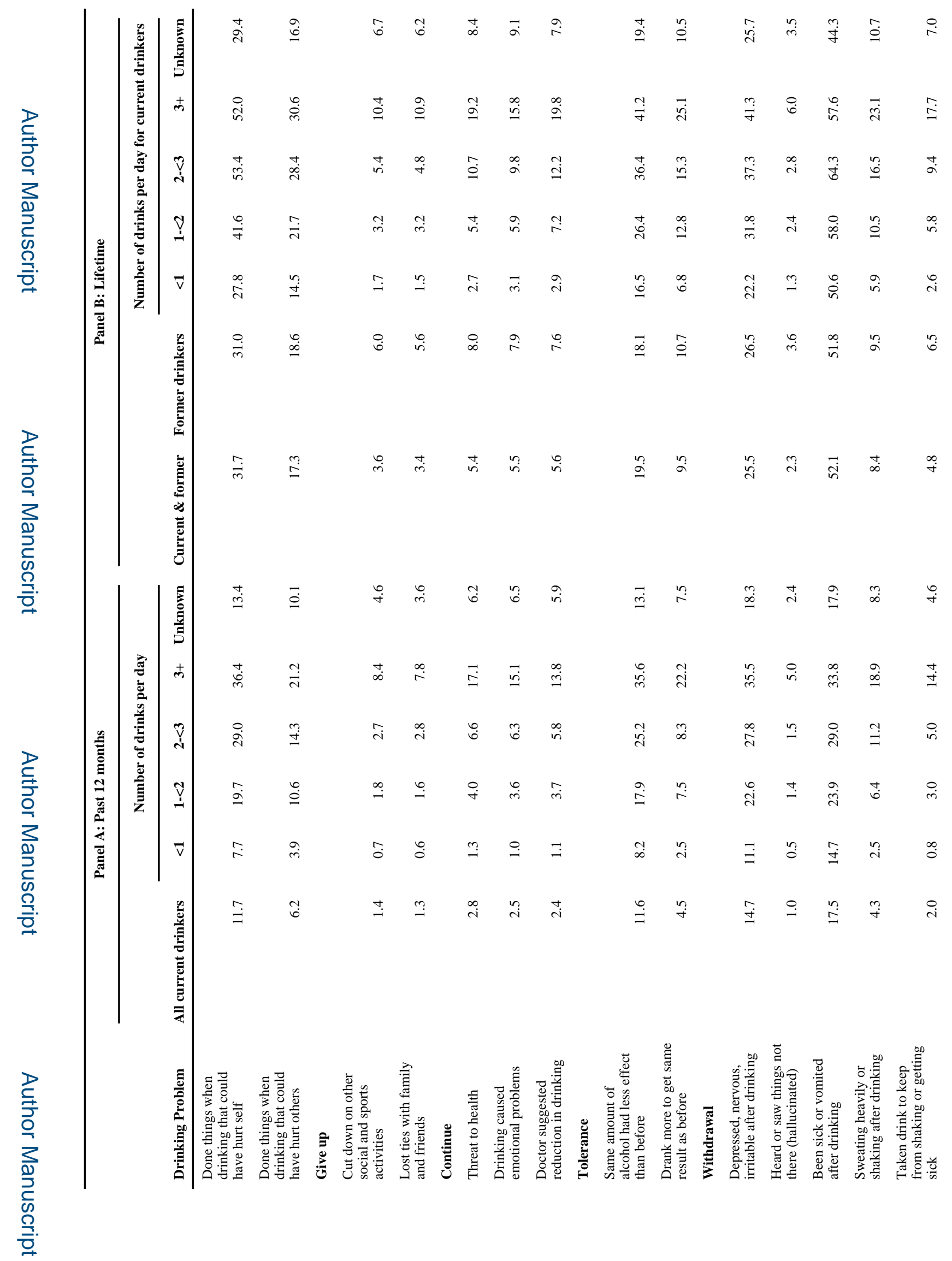

Drug Alcohol Depend. Author manuscript; available in PMC 2016 June 01. 
Rogers et al.

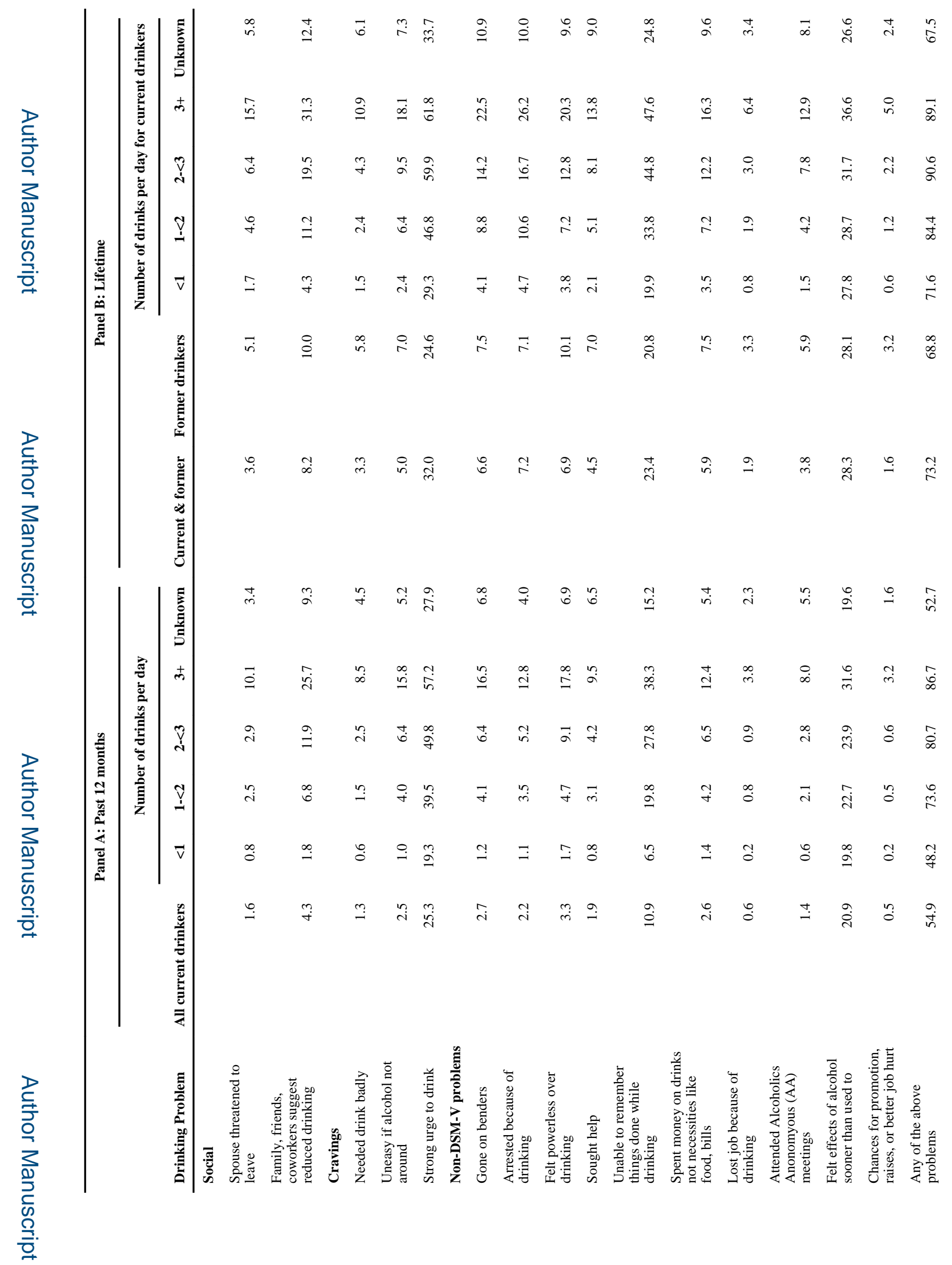

Drug Alcohol Depend. Author manuscript; available in PMC 2016 June 01. 
Rogers et al.

Page 19

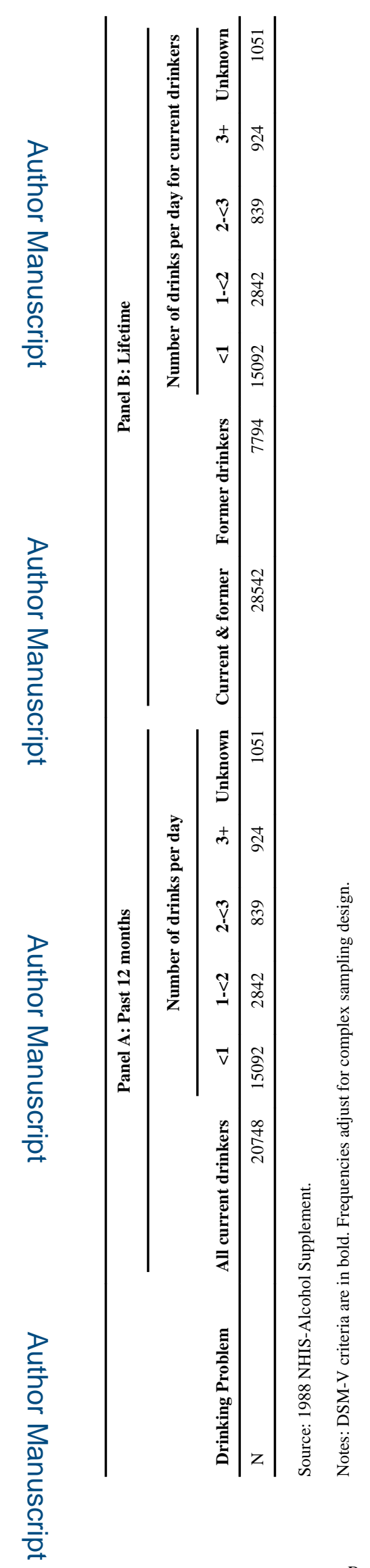

Drug Alcohol Depend. Author manuscript; available in PMC 2016 June 01. 
Table 3

Mortality risk (hazard ratios) associated with drinking problems for U.S. adults, 1988-2006.

\begin{tabular}{|c|c|c|c|c|}
\hline Drinking Problems ${ }^{a}$ & Past 12 months $b$ & 95\% CI & In lifetime $c$ & $95 \% \mathrm{CI}$ \\
\hline \multicolumn{5}{|l|}{ Larger } \\
\hline Started drinking even though hadn't intended to & 1.13 & $(1.01,1.27)$ & 1.07 & $(1.00,1.15)$ \\
\hline Difficult to stop drinking once started & 1.15 & $(1.00,1.32)$ & 1.12 & $(1.01,1.24)$ \\
\hline Kept drinking longer than intended & 1.03 & $(0.92,1.15)$ & 1.06 & $(0.99,1,14)$ \\
\hline Drinking more than intended & 1.03 & $(0.93,1.14)$ & 1.03 & $(0.96,1.10)$ \\
\hline \multicolumn{5}{|l|}{ Cut down } \\
\hline Wanted to cut down but couldn't & 1.33 & $(1.04,1.72)$ & 1.22 & $(1.04,1.43)$ \\
\hline Tried but couldn't cut down & 1.10 & $(0.83,1.46)$ & 1.11 & $(0.97,1.28)$ \\
\hline \multicolumn{5}{|l|}{ Time spent } \\
\hline Spent lots of time drinking & 1.58 & $(1.30,1.92)$ & 1.27 & $(1.15,1.41)$ \\
\hline \multicolumn{5}{|l|}{ Major role } \\
\hline Stayed away from work or gone in late $\mathrm{b} / \mathrm{c}$ hangover & 1.10 & $(0.84,1.44)$ & 1.09 & $(0.98,1.20)$ \\
\hline Got drunk rather than do things supposed to do & 1.25 & $(1.00,1.56)$ & 1.12 & $(1.01,1.24)$ \\
\hline Hangover interfered with other activities & 1.23 & $(0.99,1.54)$ & 1.13 & $(1.03,1.25)$ \\
\hline Caused problems at home, school, or work & 1.50 & $(1.14,1.99)$ & 1.32 & $(1.16,1.50)$ \\
\hline \multicolumn{5}{|l|}{ Hazard } \\
\hline Driven car after drank too much & 1.02 & $(0.89,1.17)$ & 1.01 & $(0.94,1.09)$ \\
\hline Done things when drinking that could have hurt self & 1.00 & $(0.83,1.20)$ & 1.04 & $(0.96,1.12)$ \\
\hline Done things when drinking that could have hurt others & 1.15 & $(0.92,1.45)$ & 1.05 & $(0.95,1.15)$ \\
\hline \multicolumn{5}{|l|}{ Give up } \\
\hline Cut down on other social and sports activities & 1.46 & $(1.00,2.11)$ & 1.26 & $(1.08,1.47)$ \\
\hline Lost ties with family and friends & 1.22 & $(0.87,1.73)$ & 1.32 & $(1.13,1.55)$ \\
\hline \multicolumn{5}{|l|}{ Continue } \\
\hline Threat to health & 1.44 & $(1.18,1.75)$ & 1.32 & $(1.20,1.46)$ \\
\hline Drinking caused emotional problems & 1.32 & $(1.02,1.70)$ & 1.23 & $(1.08,1.40)$ \\
\hline Doctor suggested reduction in drinking & 1.67 & $(1.32,2.12)$ & 1.33 & $(1.20,1.47)$ \\
\hline \multicolumn{5}{|l|}{ Tolerance } \\
\hline Same amount of alcohol had less effect than before & 1.18 & $(1.03,1.34)$ & 1.13 & $(1.04,1.22)$ \\
\hline Drank more to get same result as before & 1.25 & $(0.99,1.56)$ & 1.21 & $(1.09,1.34)$ \\
\hline \multicolumn{5}{|l|}{ Withdrawal } \\
\hline Depressed, nervous, irritable after drinking & 1.10 & $(0.97,1.26)$ & 1.06 & $(0.99,1.15)$ \\
\hline Heard or saw things not there (hallucinated) & 1.40 & $(0.97,2.05)$ & 1.20 & $(1.00,1.44)$ \\
\hline Been sick or vomited after drinking & 1.07 & $(0.93,1.24)$ & 0.96 & $(0.90,1.03)$ \\
\hline Sweating heavily or shaking after drinking & 1.25 & $(0.98,1.60)$ & 1.14 & $(1.02,1.26)$ \\
\hline Taken drink to keep from shaking or getting sick & 1.50 & $(1.15,1.95)$ & 1.23 & $(1.09,1.40)$ \\
\hline \multicolumn{5}{|l|}{ Social } \\
\hline Spouse threatened to leave & 1.15 & $(0.84,1.59)$ & 1.43 & $(1.25,1.64)$ \\
\hline Had family, friends, coworkers suggest reduced drinking & 1.50 & $(1.23,1.83)$ & 1.32 & $(1.20,1.46)$ \\
\hline
\end{tabular}




\begin{tabular}{|c|c|c|c|c|}
\hline Drinking Problems ${ }^{a}$ & Past 12 months $b$ & $95 \% \mathrm{CI}$ & In lifetime ${ }^{c}$ & $95 \% \mathrm{CI}$ \\
\hline Needed drink badly & 1.59 & $(1.08,2.33)$ & 1.25 & $(1.08,1.45)$ \\
\hline Uneasy if alcohol not around & 1.27 & $(1.04,1.56)$ & 1.18 & $(1.04,1.33)$ \\
\hline Strong urge to drink & 1.08 & $(0.98,1.20)$ & 1.08 & $(1.01,1.16)$ \\
\hline \multicolumn{5}{|l|}{ Non-DSM-V problems } \\
\hline Gone on benders & 1.54 & $(1.07,2.22)$ & 1.32 & $(1.16,1.52)$ \\
\hline Arrested because of drinking & 1.37 & $(1.03,1.82)$ & 1.27 & $(1.12,1.43)$ \\
\hline Felt powerless over drinking & 1.17 & $(0.89,1.54)$ & 1.17 & $(1.04,1.32)$ \\
\hline Sought help & 1.34 & $(0.87,2.05)$ & 1.23 & $(1.06,1.44)$ \\
\hline Unable to remember things done while drinking & 1.22 & $(1.04,1.45)$ & 1.12 & $(1.04,1.21)$ \\
\hline Spent money on drinks not necessities like food and bills & 1.36 & $(1.01,1.83)$ & 1.21 & $(1.06,1.37)$ \\
\hline Lost job because of drinking & 1.67 & $(1.02,2.75)$ & 1.36 & $(1.11,1.65)$ \\
\hline Attended Alcoholics Anonomyous (AA) meetings & 1.45 & $(1.04,2.02)$ & 1.38 & $(1.19,1.60)$ \\
\hline Felt effects of alcohol sooner than used to & 1.04 & $(0.93,1.17)$ & 1.07 & $(0.99,1.15)$ \\
\hline Chances for promotion, raises, or better job hurt & 1.73 & $(0.99,3.01)$ & 1.50 & $(1.25,1.84)$ \\
\hline
\end{tabular}

Source: NHIS-LMF, 1988-2006.

${ }^{a}$ Hazard ratio for each problem calculated from separate models.

${ }^{b}$ For current drinkers only. $\mathrm{N}=19,697$ (deaths=3,365).

${ }^{c}$ For current and former drinkers, $\mathrm{N}=27,491$ (deaths $=5,836$ ).

Notes: Referent for drinking problems is "none or never." Models control for sex, race/ethnicity, marital status, education, income, drinking status, smoking, BMI, and region and account for age and complex sampling design. DSM-V criteria are in bold. 
Table 4

Mortality risk (hazard ratios) associated with DSM-V criteria for U.S. adults, 1988-2006.

\begin{tabular}{|c|c|c|}
\hline DSM-V Criteria & Last 12 months ${ }^{a}$ & $95 \% \mathrm{CI}$ \\
\hline Larger & 1.06 & $(0.96,1.17)$ \\
\hline Cut down & 1.15 & $(0.86,1.54)$ \\
\hline Time spent & 1.66 & $(1.34,2.06)$ \\
\hline Major role & 1.29 & $(1.06,1.57)$ \\
\hline Hazard & 1.06 & $(0.89,1.26)$ \\
\hline Give up & 1.29 & $(0.93,1.80)$ \\
\hline Continue & 1.50 & $(1.25,1.79)$ \\
\hline Tolerance & 1.24 & $(1.08,1.42)$ \\
\hline Withdrawal & 1.38 & $(0.92,2.06)$ \\
\hline Social & 1.57 & $(1.28,1.91)$ \\
\hline Cravings & 1.12 & $(1.00,1.25)$ \\
\hline
\end{tabular}

Source: NHIS-LMF, 1988-2006.

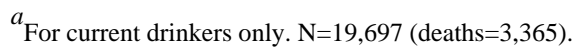

Notes: Models control for sex, race/ethnicity, marital status, education, income, drinking status, smoking, BMI, and region and account for age and complex sampling design. 
Table 5

Mortality risk (hazard ratios) of DSM diagnosis and drinking status for U.S. adults: current drinkers and abstainers, 1988-2006.

\begin{tabular}{lcc}
\hline & Model 1 (95\% CIs) & Model 2 (95\% CIs) \\
\hline DSM-V diagnosis (no SUD) & & \\
$\quad$ Mild SUD & & $1.00(0.87,1.15)$ \\
$\quad$ Moderate/severe SUD & & $1.47(1.25,1.73)$ \\
Drinking status (abstainers) & & \\
Light & $0.90(0.84,0.97)$ & $0.90(0.84,0.97)$ \\
Moderate & $1.01(0.90,1.13)$ & $0.98(0.88,1.10)$ \\
Moderate/heavy & $1.38(1.17,1.62)$ & $1.31(1.11,1.54)$ \\
Heavy & $1.47(1.25,1.73)$ & $1.35(1.16,1.56)$ \\
\hline
\end{tabular}

Source: NHIS-LMF, 1988-2006.

Notes: Referents are in parentheses. N=27,556 (6,216 deaths). Models control for sex, race/ethnicity, marital status, education, income, smoking, $\mathrm{BMI}$, and region and account for age and complex sampling design. 